\title{
Retinopathy of Valsalva and Pregnancy
}

Med Karam Saoud*, Mounsef Mahaouchi, Nisrine Mamouni, Sanae Raghay, Abdlaziz Banani

Department of Obstetrics \& Gynecology, CHU Hassan II Fes, Morocco

DOI: $10.36347 /$ sjmcr.2020.v08i03.033

| Received: 04.02.2020 | Accepted: 23.02.2020 | Published: 21.03.2020

*Corresponding author: Med Karam Saoud

\section{Abstract}

Valsalva hemorrhagic retinopathy is a rare condition, often responsible for a sharp drop in visual acuity associated with pre-macular retinal hemorrhage. Pregnancy is considered a risk factor. We report a case of Valsalva retinopathy and pregnancy, through a review of the literature we describe the pathophysiological mechanism of this condition as well as the therapeutic management modalities.

Keywords: Valsalva, pregnancy, YAG laser.

Copyright @ 2020: This is an open-access article distributed under the terms of the Creative Commons Attribution license which permits unrestricted use, distribution, and reproduction in any medium for non-commercial use (NonCommercial, or CC-BY-NC) provided the original author and source are credited.

\section{INTRODUCTION}

\section{Clinical Case}

This is a patient of 35 years, 16 Weeks of pregnancy, admitted to the ophthalmological emergencies for brutal decrease of the visual acuity of the left eye. The interrogation finds: a well followed pregnancy; vomiting at 09 week for which she was hospitalized in our departement for ten days with good clinical progress under symptomatic treatment; an influenza-like illness with coughing the last five days prior to admission.
Fundus examination revealed the presence of pre-macular retro-hyaloid hemorrhage (Figure-1). Fluorescence angiography does not show any particular vascular abnormality. A general assessment was made including a NFS, blood glucose and hemostasis report that returned normal. The diagnosis of hemorrhagic retinopathy of Valsalva is retained.

The patient benefited from a laser treatment on the hyaloid membrane in order to disperse the hematoma thus allowing its resorption.

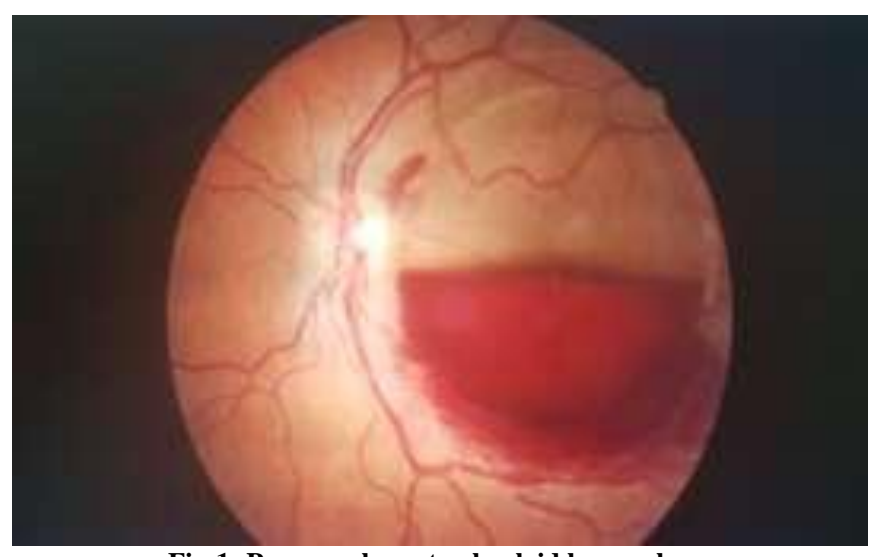

Fig-1: Pre-macular retro-hyaloid hemorrhage

\section{DISCUSSION}

Valsalva retinopathy was first described by Duane in Philadelphia in 1972 [1].

Valsalva hemorrhagic retinopathy is a rare condition, often responsible for a sharp drop in visual acuity associated with pre-macular retinal hemorrhage. Pregnancy is considered a risk factor. We report a case of Valsalva retinopathy and pregnancy, through a review of the literature we describe the pathophysiological mechanism of this condition as well as the therapeutic management modalities. 
The physiopathological mechanism of retrohyaloidal hemorrhages secondary to the Valsalva maneuver is known: there are physiological adhesions of the vitreous to the retinal vessels. The thoracic hyperpressure by inhibition in inspiration has repercussions on the cephalic and therefore retinal venous circulation. Under the effect of retinal venous hyperpressure, the adhesions pull the vessel which breaks in the vitreous cavity or in the retrohyaloid space. It is thus the vitreous traction on a vein, which causes its rupture and the blood flood [2].

The origin of retrohyaloid hemorrhage may occur in very different ways: during voluntary vomiting, during pre-labor exertion, or during intense sexual activity .... [3].

During pregnancy, there is already an increase in intra-abdominal pressure. With the efforts of vomiting and cough, the cephalic hyperpressure becomes considerable to cause the rupture of the superficial retinal capillaries [4]. Pregnancy is known as a risk factor for this condition. The prognosis is generally good with spontaneous recovery in the vast majority of cases $[4,5]$. The moment of spontaneous resorption is very variable. The use of the YAG laser may be proposed in some cases to disperse pre-retinal hemorrhages in the vitreous and thus allow a faster recovery [6].

\section{CONCLUSION}

Pregnancy is a risk factor for Valsalva retinopathy. The diagnosis should be retained after eliminating other causes of bleeding. Evolution is usually spontaneously favorable.

\section{REFERENCES}

1. Duane TD. Valsalva hemorrhagic retinopathy. Transactions of the American Ophthalmological Society. 1972;70:298-313.

2. Guigon-Souquet B, Salaun N, Macarez R, Bazin S, de la Marnierre E, Mazdou M. Hémorragie rétrohyaloïdienne secondaire à une manœuvre de Valsalva. Journal français d'ophtalmologie. 2004 Dec 1;27(10):1159-62.

3. El-Khayat AR. Valsalva haemorrhagic retinopathy in pregnancy after yoga. Case Reports. 2017 Aug 11;2017:bcr-2017.

4. Nghiem-Buffet S, Guiberteau B. Rétinopathie de Valsalva et grossesse. Images en ophtalmologie. 2009;3:86-7.

5. Fernández MG, Navarro JC, Castaño CG. Longterm evolution of Valsalva retinopathy: a case series. Journal of medical case reports. $2012 \mathrm{Dec}$ $1 ; 6(1): 346$.

6. Matonti F, Donadieu V, Hoffart L, Dornadin A, Nadeau S, Roux S, Denis D. Traitement précoce par laser Nd: YAG de la rétinopathie de Valsalva: résultats à propos de cinq cas et revue de la littérature. Journal français d'ophtalmologie. 2013 Sep 1;36(7):604-9. 